Acta Crystallographica Section D

Biological

Crystallography

ISSN 0907-4449

\section{Jean-Paul Declercq* and Christine Evrard}

Université Catholique de Louvain, Unit of Structural Chemistry (CSTR), Place Louis Pasteur 1, B-1348 Louvain-la-Neuve, Belgium

Correspondence e-mail:

declercq@chim.ucl.ac.be
C) 2001 International Union of Crystallography Printed in Denmark - all rights reserved

\title{
A twinned monoclinic crystal form of human peroxiredoxin 5 with eight molecules in the asymmetric unit
}

The monoclinic crystal form of human peroxiredoxin 5 with eight molecules in the asymmetric unit was obtained under exactly the same conditions as the tetragonal form with one molecule in the asymmetric unit, except that the latter was briefly cryosoaked with halide for derivatization. A merohedral twinning was observed, which is rather unusual in the monoclinic system and only possible with particular unit-cell dimensions. After detwinning the native and a mercury derivative, the structure was solved by the SIR method with the help of the non-crystallographic symmetry. The packing of the monoclinic and tetragonal forms are compared, with special attention to the role of bromide ions in the change of space group after crystallization. The availability of nine (eight monoclinic plus one tetragonal) independent molecules allows an analysis of the mobility. The two Cys residues implicated in the peroxide-reduction mechanism are located in rigid regions but are covered by mobile loops.

\section{Introduction}

Peroxiredoxins (PRDX) represent an emerging family of thioredoxin peroxidases which are evolutionarily conserved and have been identified in a variety of organisms ranging from prokaryotes to mammals (Chae, Robinson et al., 1994). These enzymes are able to reduce hydrogen peroxides and alkyl hydroperoxides using thioredoxin or other thiolcontaining molecules as electron donors. They are implicated in cell antioxidant protective mechanisms as well as in signal transduction.

All peroxiredoxins contain a conserved cysteine residue in the $\mathrm{N}$-terminal region of the enzyme which is oxidized by peroxides to sulfenic acid (Cys-SOH). In PRDX1 to PRDX4, which represent the so-called 2-Cys mammalian subgroup, this oxidized cysteine rapidly reacts with a conserved C-terminal cysteine of another subunit to form an intermolecular disulfide bridge. This disulfide bridge is then reduced by thioredoxin (Chae, Chung et al., 1994; Ellis \& Poole, 1997). In PRDX5, recently identified as a prototype of a new mammalian peroxiredoxin subfamily (Knoops et al., 1999), the N-terminal sulfenic acid (Cys47) reacts with a C-terminal cysteine (Cys151) of the same molecule. An intramolecular disulfide intermediate, distinct from the 2-Cys subgroup, is thus formed and can be reduced by thioredoxin subsequently (Seo et al., 2000). For PRDX6, the only known mammalian member of the 1-Cys subgroup, the mechanism by which the sulfenic acid is reduced is still to be elucidated clearly (Kang et al., 1998).

The crystal structures of PRDX1, PRDX2, PRDX5 and PRDX6 reveal that peroxiredoxins are novel members of the thioredoxin-fold superfamily (Choi et al., 1998; Declercq et al., 2001; Hirotsu et al., 1999; Schröder \& Ponting, 1998; Schröder
Received 17 July 2001

Accepted 19 September 2001

PDB Reference: Peroxiredoxin $5,1 \mathrm{~h} 4 \mathrm{o}$. 
et al., 2000; Martin, 1995). Contrary to other peroxiredoxins, no dimer formation is observed in the crystal structure of PRDX5, which has been recently solved in our laboratory in the reduced form (Declercq et al., 2001). According to the mechanism of action proposed for PRDX5, Cys47 and Cys151 are implicated in the peroxide reduction activity by forming an intramolecular disulfide intermediate under oxidizing conditions. However, in the reduced form of PRDX5, these two cysteines are located too far apart to interact with each other without large conformational changes. Moreover, the presence of a benzoate ion close to the active-site pocket suggests that PRDX5 could present a broader activity towards reactive oxygen species compared with other peroxiredoxins or antioxidant enzymes.

In this paper, we report the monoclinic crystal structure of PRDX5 in its reduced form. The structure has been determined by the SIR method and refined at $1.95 \AA$ resolution. A comparison with the first crystal structure of PRDX5 (Declercq $e t$ $a l ., 2001)$, which was crystallized in the tetragonal system, is also presented.

\section{Experimental}

\subsection{Crystallization}

The expression and purification of human PRDX5 have previously been described (Knoops et al., 1999; Declercq et al., 2001). The crystals of the $6 \times$ Histagged molecule were grown under reducing conditions by hanging-drop vapour diffusion at $291 \mathrm{~K}$, with the well solution $(500 \mu \mathrm{l})$ consisting of $1.6 \mathrm{M}$ ammonium sulfate and $0.2 M$ potassium sodium tartrate as precipitant, $0.1 \mathrm{M}$ sodium citrate buffer $\mathrm{pH}$ 5.3, $10 \mathrm{mM}$ 1,4-dithio-DL-threitol as antioxidant and $0.02 \%(w / v)$ sodium azide. The hanging drop was formed by mixing $2 \mu \mathrm{l}$ of the protein solution $\left(10 \mathrm{mg} \mathrm{ml}^{-1}\right)$ with $2 \mu \mathrm{l}$ of the well solution. As discussed in $\S 3$, this monoclinic crystal form is obtained using exactly the protocol previously described for the tetragonal form characterized by one molecule only in the asymmetric unit (Declercq et al., 2001).

\subsection{Data collection and processing}

Before data collection, the crystals were cryosoaked for about $30 \mathrm{~s}$ in a solution identical to the crystallization buffer but containing $20 \%(v / v)$ glycerol

Figure 1 and flash-cooled at $100 \mathrm{~K}$. One cryocooled crystal was used for data collection at $1.95 \AA$ resolution on the synchrotron beamline BM30A at ESRF, using a MAR345 image-plate detector. The diffraction images were easily indexed with DENZO (Otwinowski \& Minor, 1997) in a $C$-centered orthorhombic lattice with unit-cell parameters $a=131, b=250$, $c=67 \AA$, but all the efforts to merge the data with SCALE-

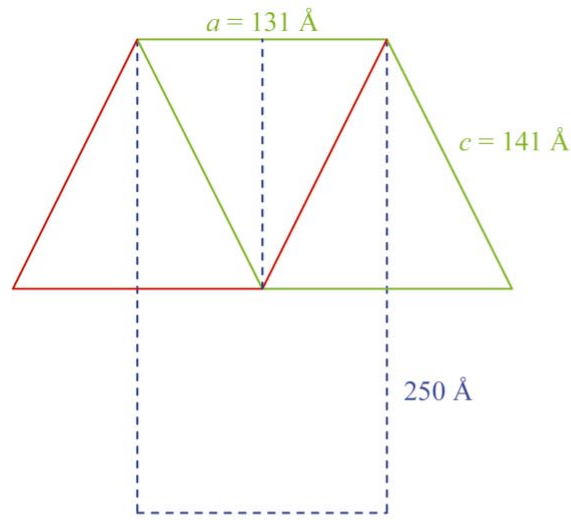

(a)

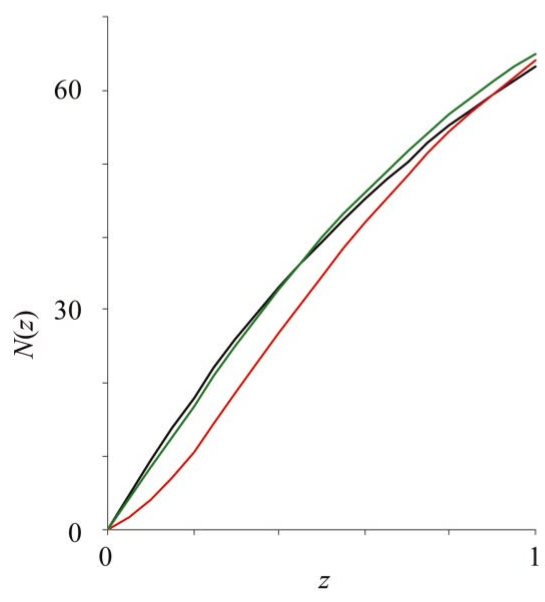

(c)

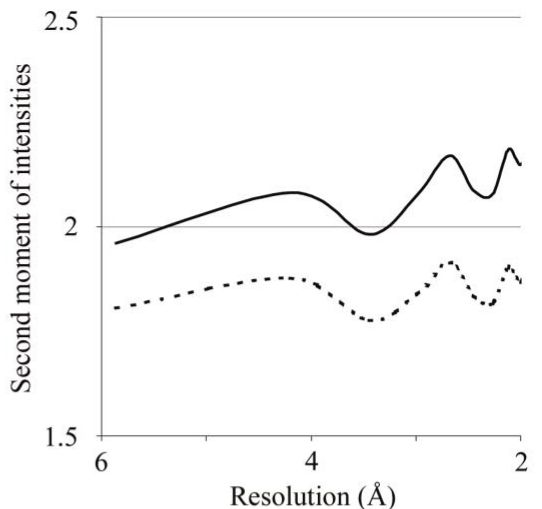

(b)

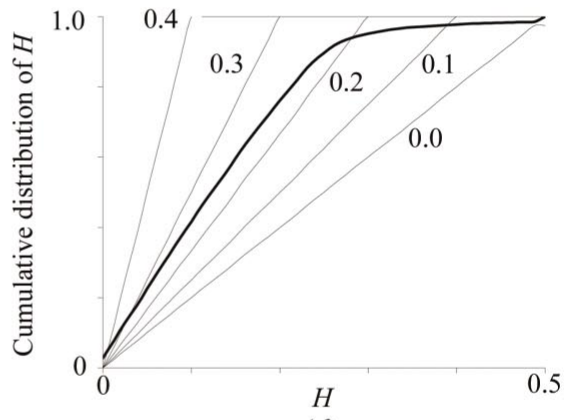

(d)

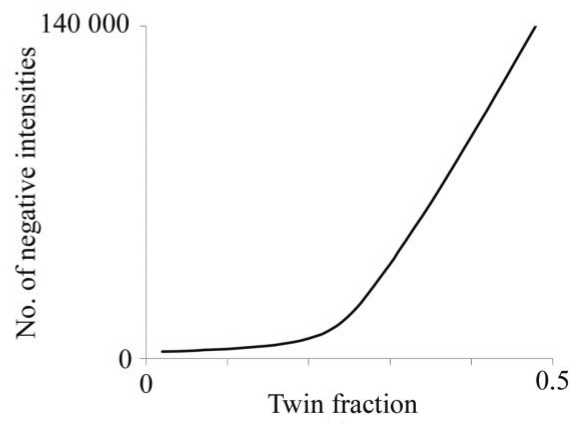

(e)

(a) Projection of unit cells on the $a c$ plane. The $a$ axis is horizontal. The length of the $b$ axis (perpendicular to the projections) is about $66 \AA$ in all cases. The monoclinic unit cell is green. The twinning is produced by the superposition of the red cell. In case of a perfect twinning (twin fraction $50 \%$ ), the orthorhombic Laue group $\mathrm{mmm}$ would be perfectly simulated, with a $C$-centered lattice represented in dashed blue. The tetragonal primitive cell described by Declercq et al. (2001) is also dashed blue and corresponds to one half of the other cells in the direction of $a$. (b) Second moment of the intensities $\left(\left\langle I^{2}\right\rangle /\langle I\rangle^{2}\right)$ as a function of resolution before detwinning (dotted line) and after detwinning (full line). The expected values are 2.0 for non-twinned data and 1.5 for a perfect twin. (c) Cumulative intensity distribution of acentric data: $N(z)$ is the percentage of reflections with $I /\langle I\rangle$ less or equal to $z$. Theoretical values are black; observed data are red before detwinning and green after detwinning. $(d)$ Cumulative distribution of $H=\left\langle\left|I_{\text {obs }}(1)-I_{\text {obs }}(2)\right| /\left[I_{\text {obs }}(1)+I_{\text {obs }}(2)\right]\right\rangle$ as a function of $H$ (Yeates, 1997). $I_{\text {obs }}(1)$ and $I_{\text {obs }}(2)$ are the intensities of two reflections related by the twin operator before detwinning. The thick line shows the experimental data and the thin lines show the expected values for various twin fractions. (e) Number of negative intensities after detwinning as a function of the twin fraction. 
PACK (Otwinowski \& Minor, 1997) were unsuccessful $\left(R_{\text {sym }}>20 \%\right)$. Finally, the data were successfully indexed in the less symmetric monoclinic space group $P 2_{1}$, with unit-cell parameters $a=130.79, b=66.49$, $c=141.24 \AA, \beta=117.54^{\circ}$ and eight molecules in the asymmetric unit. The authors are fully aware of the possible indexing of any true $C 222_{1}$ lattice as monoclinic $P 2_{1}$, but a true orthorhombic lattice would respect the Laue group $m m m$ and that is not the case here. Fig. 1(a) shows the relationship between the pseudo- $C$-centered orthorhombic cell and the correct monoclinic cell and also suggests some resemblance (discussed in the next paragraphs) to the previously described tetragonal cell (Declercq et al., 2001). Some statistics of data collection and processing are presented in Table 1 .

\subsection{Structure solution and refinement}

At the time of attempting the crystal structure solution, the structure of the tetragonal form was not yet available and only two PRDX structures were at our disposal (Choi et al., 1998; Hirotsu et al., 1999) with only $\sim 10 \%$ sequence homology. Since all the attempts to solve the structure by molecular replacement using these models failed, two heavy-atom derivatives (methylmercury chloride, $\mathrm{HgCH}_{3} \mathrm{Cl}$; potassium iodide, KI) were collected using a rotating-anode generator. Using SHELXS97 (Sheldrick et al., 1993), it was possible to find several different interpretations of the isomorphous difference Patterson of the mercury derivative. Each of these interpretations was self-consistent in the sense that the necessary interatomic vectors were observed in the Patterson, but it was not possible to merge any of these interpretations with the others. Furthermore, none of the difference Fourier syntheses phased with the interpretations of this derivative were able to produce heavy-atom positions of the iodide derivative consistent with its isomorphous difference Patterson function. This observation suggested that the particular dimensions of the unit cell could allow the perfect merohedral twinning illustrated in Fig. 1(a), which could also explain the pseudo- $C$ centered orthorhombic lattice. Indeed, in the case of a perfect hemihedral twinning (twin fraction $\simeq 50 \%$ ), one could expect that the merging of the data in an orthorhombic lattice would proceed nicely; this is not at all the case for the native data set (orthorhombic $R_{\mathrm{sym}}>20 \%$ ), but seems much more likely for the two derivatives (orthorhombic $R_{\text {sym }}=0.094$ and 0.095 compared with the monoclinic values of 0.069 and 0.087 , respectively). The presence of twinning can also be detected by an analysis of the second moment of intensities: a value of 2.0 is expected in the absence of twinning and of 1.5 in the case of a perfect twin. Fig. 1(b) shows this second moment as a function of resolution for the native data and the experimental values close to 1.8 suggest a partial twinning. The same conclusion is drawn from the observed cumulative distribution of intensities presented in Fig. 1(c). The native data set was thus analysed with the program DETWIN of the CCP4 suite (Collaborative Computational Project, Number 4, 1994) in order to determine the twin fraction. A plot of the observed distribution of $H$ (Yeates, 1997) compared with the expected distribution for various twin fractions (Fig. 1d) and a plot showing the number of negative intensities after detwinning as a function of the twin fraction (Fig. 1e) allowed an estimation of the twin fraction as $\sim 0.24$. A similar analysis applied to the derivatives provided much less favourable estimates ( 0.42 for $\mathrm{HgCH}_{3} \mathrm{Cl}$ and 0.46 for $\mathrm{KI}$ ) as could be expected from the orthorhombic $R_{\mathrm{sym}}$ values. The twinning illustrated in Fig. 1(a) corresponds to two monoclinic cells with coincident $b$ (and $a$ ) axes, but in opposite directions in such a way that the monoclinic Laue group $2 / m$ remains perfectly valid. A similar analysis in reciprocal space (not shown) allowed definition of the twin operator as $-h,-k, h+l$. After detwinning, $5 \%$ of the reflections were flagged for use in $R_{\text {free }}$ calculations (Brünger, 
1992). The anomalous signals of the derivatives were lost and the statistics (Table 1) clearly indicate that the iodide derivative was completely unusable, which is not very surprising since its twin fraction is very close to $50 \%$. Even after detwinning, no valuable molecular-replacement solution was obtained.

A self-rotation function applied to the native data (Fig. 2) clearly indicates a non-crystallographic fourfold axis in the

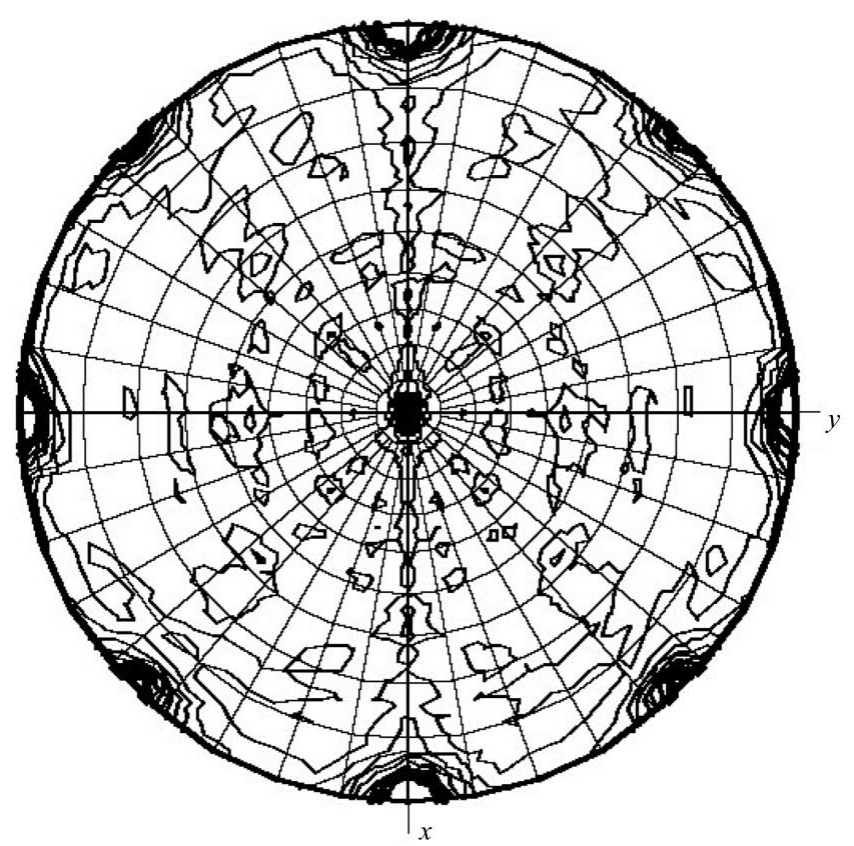

(a)

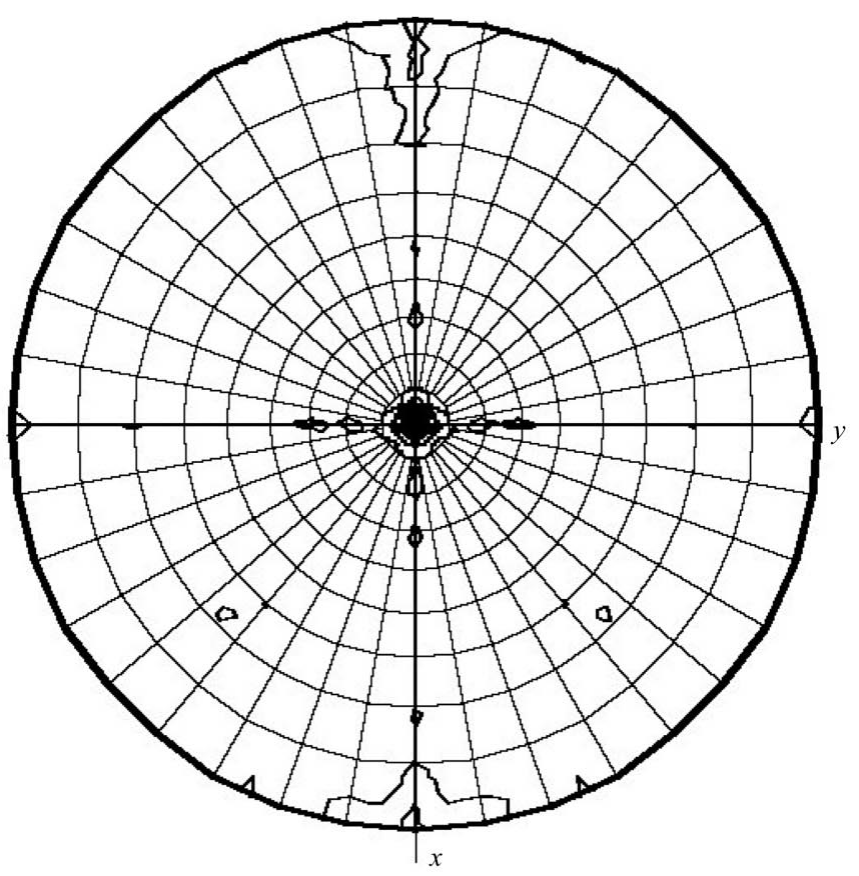

(b)

Figure 2

The self-rotation function after detwinning, contoured at $1 \sigma$ and represented in orthonormal axes with $x$ parallel to $a$ and $z$ parallel to $c^{*}$. (a) Twofold NCS rotation; (b) fourfold NCS rotation. direction of $c^{*}$ and four twofold axes perpendicular to the latter, one of which coincides with the crystallographic $2_{1}$ axis, suggesting a non-crystallographic symmetry corresponding to the point group 422. A native Patterson function at $4.0 \AA$ resolution shows the largest non-origin peak (height $10.7 \sigma$ ) at the fractional position $(0.50,0.05,0.00)$, suggesting an approximate pure translation of half a cell in the direction of a. A posteriori, it will be seen that this translation corresponds to one complete unit cell in the tetragonal system (see Fig. 1a).

The isomorphous difference Patterson of the mercury derivative was interpreted at $3.0 \AA$ resolution by SHELXS-97 (Sheldrick et al., 1993) and provided eight heavy-atom positions. Their consistency was checked using the program MLPHARE (Otwinowski, 1991) from the CCP4 suite (Collaborative Computational Project, Number 4, 1994): after randomly removing three $\mathrm{Hg}$ atoms at a time, single isomorphous replacement (SIR) phases were computed with the remaining five $\mathrm{Hg}$ atoms and each time the first three new peaks appearing in an isomorphous difference synthesis corresponded to the three missing heavy atoms. Interestingly, it was also noticed that it was possible to find four pairs of heavy-atom positions related by a very approximate translation corresponding to $a / 2$. Since only one derivative lacking anomalous signal was usable, the correct enantiomer could not be defined at this stage and all the subsequent steps were run for both enantiomers until the quality of the maps allowed selection of the correct one. SIR phases were then computed at $2.5 \AA$ using the eight mercury positions and the resulting phases were refined by solvent flattening in $D M$ (Cowtan, 1994), assuming a solvent content of $61 \%$. After tracing the bones using MAPMAN (Kleywegt \& Jones, 1996), it was not possible to recognize any secondary structure nor to trace any fragments, but very approximate contours were distinguishable for the eight molecules and we noticed that the eight $\mathrm{Hg}$ atoms of the derivative were located on the surface of the eight supposed molecules. Using the graphics, each of the centres of the eight molecules was roughly evaluated and associated with the coordinates of the $\mathrm{Hg}$ atom lying on their surfaces. This exercise provided eight pairs of coordinates, four of them being correlated to the four remaining ones by a single $a / 2$ translation; each group of four pairs of coordinates should be related by a NCS fourfold axis directed along $c^{*}$ but associated with unknown translation parts. A careful examination of the projection along $c^{*}$ of these coordinates allowed identification of the position of the approximate fourfold axes. A simple spherical mask with a radius of $17 \AA$ was built around one molecule and allowed to define the rotation and translation matrices corresponding to the seven other molecules. The NCS fourfold axis looked like a screw axis and thus the molecules do not obey the 422 point group. These initial matrices were refined (with difficulty) using $R A V E$ (Kleywegt $\&$ Jones, 1994) in a mixture of manual and automatic modes and correlation coefficients of between 0.3 and 0.4 were finally reached. The availability of these matrices allowed re-running of $D M$ including the NCS, an automatic mask tracing and increasing the resolution to $1.95 \AA$. Although this mask was not very convincing, the molecular contours were much better 
defined and some secondary-structural elements began to appear in the resulting map. A new mask was thus traced by hand and reintroduced for additional cycles of phase refinement in $D M$, during which all the NCS correlation coefficients refined to values above 0.9 . The new map at $1.95 \AA$ was excellent and was submitted to $A R P / w A R P$ (Perrakis et al., 1999) for automatic tracing: 200 cycles in 'warpNtrace' mode resulted in 1235 out of 1288 residues in 14 chains and most of the side chains were correctly traced in 'side-dock' mode. A manual examination with the molecular-graphics program $O$ (Jones et al., 1991) showed only one misinterpreted fragment of about 20 residues (sequentially displaced by one residue) at the N-terminal end of one chain. Most of the missing fragments were easily completed and some manual adjustments were performed with $O$. For two chains ( $B$ and $H$ ) the region around residue 20 could only be traced after several cycles of refinement using REFMAC5 (Murshudov et al., 1997). The $\mathrm{N}$-terminal His tails were not observed. NCS restraints were applied throughout the refinement. As was also the case in the tetragonal crystal form of PRDX5 (Declercq et al., 2001), a benzoate ion was observed close to the cleft containing the active site (Cys47) in each of the eight molecules and was incorporated in the model. Solvent molecules were progressively introduced by $A R P / w A R P$ and resulted in an $R$ value of $0.193\left(R_{\text {free }}=0.227\right)$ for all available data. Different twin fractions were tested and a value of 0.203 yielded slightly better results $\left(R=0.191, R_{\text {free }}=0.223\right)$. After some minor adjustments, a significant improvement was reached by refining the mean-square displacements of rigid bodies, each molecule being defined as a different TLS group. The final $R$ value is $R=0.165\left(R_{\text {free }}=0.190\right)$ for a model containing 1213 solvent molecules.

\section{Results and discussion}

\subsection{Quality of the structure and general description}

The structure refined at $1.95 \AA$ resolution contains eight molecules labelled $A$ to $H$ in the asymmetric unit. The 161 residues of the eight molecules are well defined in the electron density, but the N-terminal His tails and the short linkers are not observed in any of them. An overall coordinate error of $0.11 \AA$ is estimated based on (free) $R$ value or on maximum likelihood. The analysis of the Ramachandran plot (not shown) computed with the program PROCHECK (Laskowski et al., 1993) shows that $89.9 \%$ of the non-glycine residues are in most favoured regions and there are no residues in disallowed regions. One residue (Thr150) systematically lies in a generously allowed region in the eight molecules and will be discussed in the following paragraphs. The overall structure is characterized by the presence of a thioredoxin fold consisting of a four-stranded $\beta$-sheet and three flanking $\alpha$-helices (Martin, 1995). Each molecule comprises three additional $\alpha$-helices and three $\beta$-strands, one of which is associated with the thioredoxin $\beta$-sheet to form a fifth strand, while the two remaining ones form an additional two-stranded $\beta$-sheet in the $\mathrm{N}$-terminal part of the molecule. Since the overall folding is very similar to that described for the tetragonal crystal form of PRDX5 (Declercq et al., 2001), it will not be discussed further.

\subsection{The packing of the molecules and comparison with the tetragonal crystal form}

The crystals of this twinned monoclinic form with eight molecules in the asymmetric unit were grown in rigorously similar conditions to the tetragonal form with one molecule in the asymmetric unit described by Declercq et al. (2001). The only difference concerns the cryosoaking conditions before flash-cooling and data collection. In the monoclinic form, the crystal was soaked for $30 \mathrm{~s}$ in a solution identical to the crystallization buffer containing $20 \%(v / v)$ glycerol, while the tetragonal form was produced by cryosoaking for the same duration in the same solution but containing $1.0 \mathrm{M}$ sodium bromide in order to apply the MAD method after derivatization by short cryosoaking with halides (Dauter et al., 2000; Dauter \& Dauter, 2001). The same experiments were reproduced many times and systematically led to the same results: the non-twinned tetragonal form, which diffracts to higher resolution ( $1.5 \AA$ ) and contains only one molecule in the asymmetric unit, is only obtained in the presence of sodium bromide. We have also checked that native crystals mounted in capillaries at room temperature, without cryoprotectant, correspond to the monoclinic form. The transformation leading to a better ordering must thus occur in the crystal without introducing dislocations. This subtle transformation is probably rendered possible by a rather loose packing (solvent content $\sim 67 \%, V_{\mathrm{M}}=3.75 \AA^{3} \mathrm{Da}^{-1}$ ). A similar change of space group and system from orthorhombic to hexagonal upon soaking in $\mathrm{NaBr}$ was described by Dauter et al. (2001) for pepstatin-insensitive carboxyl proteinase (PCP). Since this transformation occurs in the previously formed crystal, the packing in both cells should be approximately similar. The dimensions of the monoclinic and tetragonal cells are compared in Fig. 1. The $b$ dimensions of both cells are roughly similar ( $66 \AA$ ), while the $a$ dimension of the monoclinic cell is twice as large (131 $\AA$ ) and thus corresponds to two tetragonal cells in that direction. In the monoclinic form, we observe an approximate translation of a half unit-cell in the direction of $a$. The length of the $c$ dimension $(123 \AA)$ in the tetragonal cell corresponds to $1 / c^{*}$ of the monoclinic cell. The similarity of the two packings was checked by building eight symmetry-related molecules in the tetragonal system: four molecules were generated by the application of the crystallographic $4_{1}$ screw axis and a duplicate of these four copies was translated by one unit cell in the $a$ direction. We then looked for the best superposition of the $\mathrm{C}^{\alpha}$ atoms of this ensemble of eight molecules on the corresponding atoms of the eight independent molecules in the monoclinic form. This superposition was best performed applying a nearly pure translation with fractional components close to $(x-1 / 4, y-0.31, z-1 / 8)$ and resulted in an r.m.s. deviation of $2.4 \AA$ between the 1288 $\mathrm{C}^{\alpha}$ atoms. The result of the superposition is shown in Fig. 3. The translations in the $x$ and $z$ directions bring a $2_{1}$ screw axis of the tetragonal cell into coincidence with the monoclinic 
screw axis and the translation in the $y$ direction corresponds to a free choice of origin in the monoclinic cell. The overall packings are thus extremely similar. After this best global superposition, we tried to independently realign each of the eight molecules. Individual rotations ranging from 1.5 to $11.1^{\circ}$ were necessary and the eight r.m.s. values were $0.275,0.455$, $0.244,0.193,0.220,0.197,0.272,0.512 \AA$ for the best superpositions of the $\mathrm{C}^{\alpha}$ atoms of chains $A$ to $H$, respectively. At this stage, it seemed interesting to analyse the exact role of the bromide ions in the packing of the tetragonal form. There are five bromide ions in this structure. All of them form hydrogenbond interactions with one or more solvent molecules. The other contacts involve amide $\mathrm{N}$ atoms from the main chain and from a glutamine side chain, a hydroxyl group from a serine and ionic pairs with the amine groups of two lysines. As shown in Table 2, only two of these anions (Br301 and Br303) are involved in specific intermolecular contacts. In the tetragonal form, the contacts mediated by the bromide ions always link one molecule with the symmetry-related molecule (labelled \# in Table 2) obtained by application of the $4_{1}$ operation $(1 / 2-$

\section{Table 2}

Intermolecular contacts $(\AA)$ mediated by bromide ions in the tetragonal form ('Tetra') and comparison with the simulated corresponding distances in the monoclinic form.

$A-B, B-C$ etc. mean that the simulated bromide ion would lie at the interface between molecules $A$ and $B, B$ and $C$ etc. The \# symbol indicates that the atom belongs to the second molecule. In the tetragonal form, the second molecule is generated by applying the symmetry operation $(1 / 2-y$, $1 / 2+x, 1 / 4+z)$. In the case of $\mathrm{Br} 301$, the contacts with the side chain of Glu60 represent unfavourable contacts which are not present in the tetragonal form but appear in the simulation of the monoclinic form.

\begin{tabular}{|c|c|c|c|c|c|c|c|c|c|c|}
\hline & & Tetra & $A-B$ & $B-C$ & $C-D$ & $D-E$ & $E-F$ & $F-G$ & $G-H$ & $H-A$ \\
\hline \multirow[t]{5}{*}{ Br301 } & Ser118 $\mathrm{O}^{\gamma}$ & 3.20 & 3.10 & 3.20 & 3.03 & 2.94 & 3.01 & 2.92 & 3.13 & 3.07 \\
\hline & Lys63 $\mathrm{N}^{\zeta} \#$ & 3.42 & 3.98 & 4.84 & 4.76 & 5.07 & 3.50 & 4.83 & 4.11 & 4.77 \\
\hline & Lys93 $\mathbf{N}^{\zeta} \#$ & 3.40 & 6.17 & 4.94 & 3.40 & 5.23 & 5.23 & 3.96 & 6.40 & 5.58 \\
\hline & Glu60 $\mathrm{O}^{\varepsilon 1} \#$ & 4.43 & 1.75 & 3.79 & 4.35 & 4.32 & 4.27 & 4.64 & 2.93 & 4.70 \\
\hline & Glu60 $\mathrm{O}^{\varepsilon 2} \#$ & 3.80 & 2.60 & 3.02 & 3.26 & 3.09 & 3.03 & 3.17 & 1.78 & 3.86 \\
\hline \multirow[t]{2}{*}{ Br303 } & Leu149 N & 3.36 & 3.28 & 3.25 & 3.30 & 3.33 & 3.27 & 3.34 & 3.30 & 3.29 \\
\hline & $\mathrm{G} \ln 133 \mathrm{~N}^{\varepsilon 2} \#$ & 3.45 & 3.63 & 4.25 & 3.99 & 3.29 & 3.84 & 4.13 & 3.81 & 4.92 \\
\hline
\end{tabular}
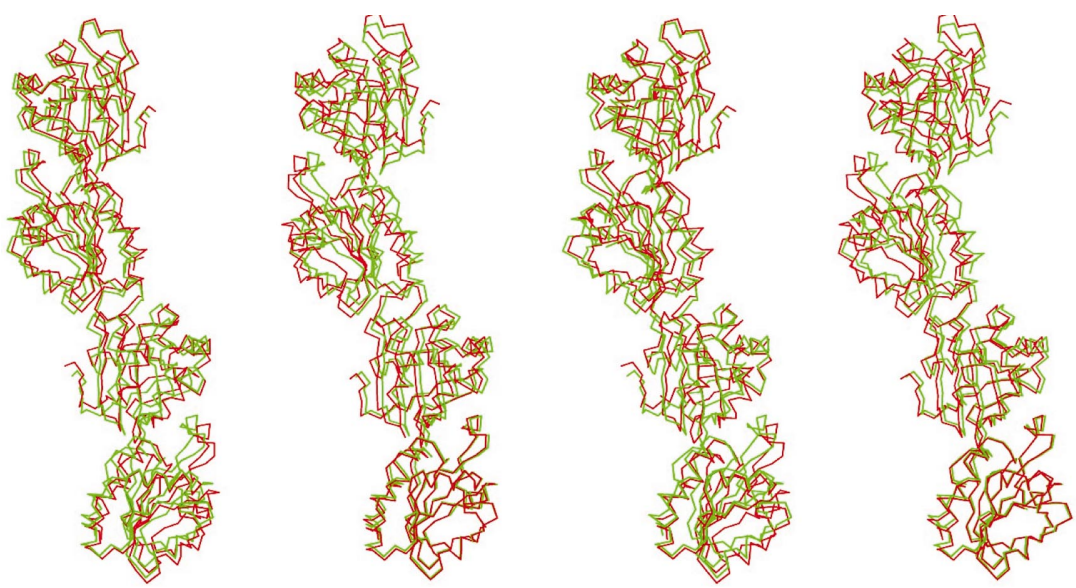

Figure 3

Stereoscopic view projected along $b$ showing a global superposition of the eight molecules present in the asymmetric unit of the monoclinic crystal form (red) with eight symmetryrelated molecules of the tetragonal form (green). The $a$ axis is horizontal and $c^{*}$ is vertical. The $A$ chain is on the bottom left of the figure and the $H$ chain on the top right. The figure was produced with $O$ (Jones et al., 1991). $y, 1 / 2+x, 1 / 4+z)$. In this way, $\mathrm{Br} 301$ becomes the bridge (y) belonging to the same symmetry-related bridge formed by $\mathrm{Br} 303$ between Leu149 $\mathrm{N}$ of the first molecule and $\mathrm{G} \ln 133 \mathrm{~N}^{\varepsilon 2}$ of the second molecule. The consequence is that the approximate non-crystallographic fourfold screw axis observed in the monoclinic form (see Experimental ction) becomes a crystallographic $4_{1}$ axis in the presence of contacts of these two bromide ions in the monoclinic cell should be without reorganization. For this purpose, we have superposed the molecule of the tetragonal form accompanied by its bromide ions on each of the eight molecules of the meclinic form and computed the distances between these ions and the relevant atoms of the molecules. It can most cases some favourable intermolecular contacts present in the tetragonal form are lost in the monoclinic form. At the same time, short prohibited contacts (electrostatic repulsion) between Br301 and the side chain of Glu60 of the neighbouring molecule can be observed. These short contacts have disappeared in the tetragonal arrangement.

\subsection{Analysis of the mobility}

As explained in $\$ 3.2$, the eight molecules were individually aligned on the unique molecule present in the tetragonal form. This superposition of nine molecules allows estimation of the mobility along the chain. For each of the 161 residues, 36 distances between the $\mathrm{C}^{\alpha}$ atoms can thus be computed, as well as their r.m.s. value (program LSQMAN; Kleywegt $\&$ Jones, 1997). The results are presented in Fig. 4. A priori, one might expect a correlation between this mobility and the atomic displacement parameters but, as shown in Fig. $4(a)$, such a correlation is not observed. The largest mobility occurs in the loop (16-21) between the two $\beta$-strands $\beta 1$ and $\beta 2$ forming the additional two-stranded $\beta$-sheet which is not part of the thioredoxin fold. In this region, the conformation of two chains $(B$ and $H)$ is very different from the seven remaining ones. In Figs. 4(b) and 4(c), the chain has been coloured according to the observed mobility. It can be seen that the central region of the molecule is extremely stable. On the same figure, the positions of the three Cys residues are indicated. All of them appear in rigid regions. The proposed peroxide-reduction mechanism (Seo et al., 2000) of the enzyme 
involves the formation of an intramolecular disulfide intermediate between Cys47 and Cys151 which can be further reduced by thioredoxin; the same authors have shown that the mutation of Cys72 had no effect on activity. According to Declercq et al. (2001), it is likely that large conformational changes must occur in PRDX5 upon oxidation. Surprisingly, Cys47 and Cys151 do not present any significant mobility, but they are covered by two flexible loops (113-116 and 144-146). These loops seem to obstruct the way between the two Cys residues and it is possible that their movement is the first step towards bringing together the two Cys residues. Thr150, one of the neighbours of Cys151, is characterized by unusual $\varphi, \psi$ values (mean values for the nine molecules: $-139,-97^{\circ}$ ) corresponding to a generously allowed region. Since similar values are observed in the nine molecules, this peculiar conformation in a stable region surrounding one of the catalytic Cys residues is certainly not fortuitous and may contribute to impose some specific orientation to Cys151.

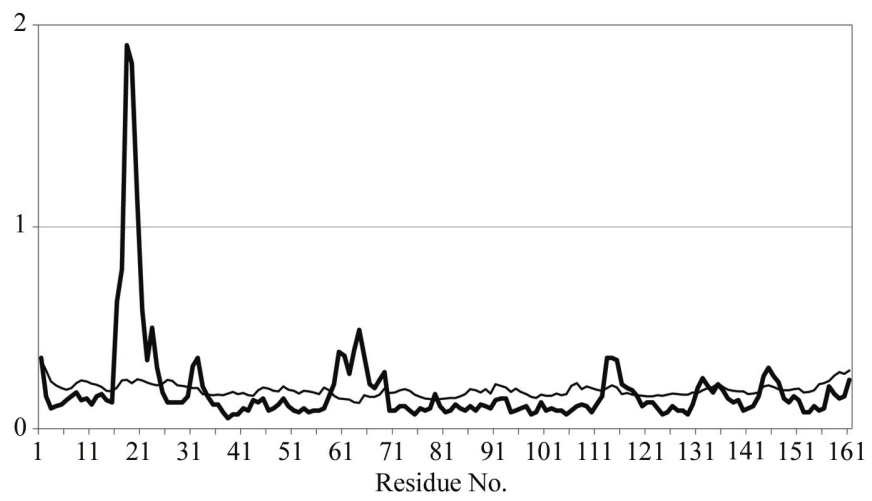

(a)

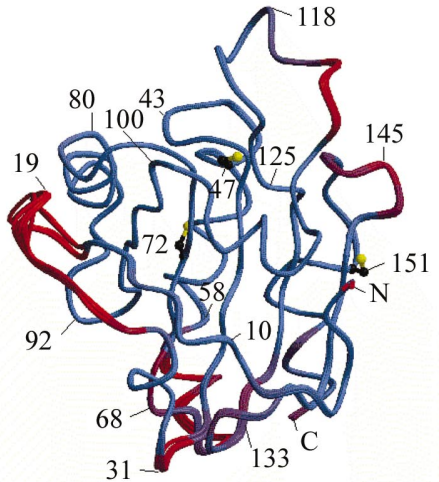

(b)

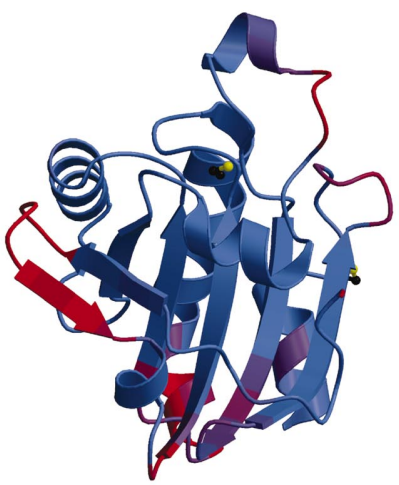

(c)

\section{Figure 4}

(a) R.m.s. distances $(\AA)$ between the $\mathrm{C}^{\alpha}$ atoms of the nine superposed PRDX5 molecules (thick line) compared with the mean atomic displacement parameters $U\left(\AA^{2} ; U=B / 8 \pi^{2}\right)$ of the $C^{\alpha}$ atoms of the eight molecules present in the monoclinic form (thin line). (b) Superposition of the nine main-chain traces coloured as a function of the r.m.s. distances between the $\mathrm{C}^{\alpha}$ atoms, from blue for the most rigid regions to red for the most flexible ones. (c) Ribbon diagram of one selected chain (chain $F$ ) coloured as in $(b)$ and showing the secondarystructural elements. In $(b)$ and $(c)$, the side chains of the Cys residues are represented. The orientations are exactly the same as Fig. 1 of Declercq et al. (2001). (b) and (c) were produced using MOLSCRIPT (Kraulis, 1991) and RASTER3D (Merritt \& Bacon, 1997).
The authors are indebted to the 'Fonds de la Recherche Fondamentale Collective' (Belgium) and to the 'Fonds National de la Recherche Scientifique' (Belgium) for financial support. We wish to thank Professor Bernard Knoops for providing the sample of purified PRDX5 and Dr Eric Fanchon for his help on the ESRF BM30 beamline.

\section{References}

Brünger, A. T. (1992). Nature (London), 355, 472-475.

Chae, H. Z., Chung, S. J. \& Rhee, S. G. (1994). J. Biol. Chem. 269, 27670-27678.

Chae, H. Z., Robinson, K., Poole, L. B., Church, G., Storz, G. \& Rhee, S. G. (1994). Proc. Natl Acad. Sci. USA, 91, 7017-7021.

Choi, H. J., Kang, S. W., Yang, C. H., Rhee, S. G. \& Ryu, S. E. (1998). Nature Struct. Biol. 5, 400-406.

Collaborative Computational Project, Number 4 (1994). Acta Cryst. D50, 760-763.

Cowtan, K. (1994). Jnt CCP4/ESF-EACBM Newsl. Protein Crystallogr. 31, 24-28.

Dauter, Z. \& Dauter, M. (2001). Structure, 9, R21-R26.

Dauter, Z., Dauter, M. \& Rajashankar, K. R. (2000). Acta Cryst. D56, 232-237.

Dauter, Z., Li, M. \& Wlodawer, A. (2001). Acta Cryst. D57, 239-249.

Declercq, J. P., Evrard, C., Clippe, A., Vander Stricht, D., Bernard, A. \& Knoops, B. (2001). J. Mol. Biol. 311, 751-759.

Ellis, H. R. \& Poole, L. B. (1997). Biochemistry, 36, 13349-13356.

Hirotsu, S., Abe, Y., Okada, K., Nagahara, N., Hori, H., Nishino, T. S. \& Hakoshima, T. (1999). Proc. Natl Acad. Sci. USA, 96, 12333 12338.

Jones, T. A., Zou, J.-Y., Cowan, S. W. \& Kjeldgaard, M. (1991). Acta Cryst. A47, 110-119.

Kang, S. W., Baines, I. C. \& Rhee, S. G. (1998). J. Biol. Chem. 273, 6303-6311.

Kleywegt, G. J. \&. Jones, T. A. (1994). Proceedings of the CCP4 Study Weekend. From First Map to Final Model, edited by S. Bailey, R. Hubbard \& D. Waller, pp. 59-66. Warrington: Daresbury Laboratory.

Kleywegt, G. J. \& Jones, T. A. (1996). Acta Cryst. D52, 826-828.

Kleywegt, G. J. \& Jones, T. A. (1997). Methods Enzymol. 277, 525545 .

Knoops, B., Clippe, A., Bogard, C., Arsalane, K., Wattiez, R., Hermans, C., Duconseille, E., Falmagne, P. \& Bernard, A. (1999). J. Biol. Chem. 274, 30451-30458.

Kraulis, P. J. (1991). J. Appl. Cryst. 24, 946-950.

Laskowski, R. A., MacArthur, M. W., Moss, D. S. \& Thornton, J. M. (1993). J. Appl. Cryst. 26, 283-291.

Martin, J. L. (1995). Structure, 3, 245-250.

Merritt, E. A. \& Bacon, D. J. (1997). Methods Enzymol. 277, 505-524. Murshudov, G. N., Vagin, A. A. \& Dodson, E. J. (1997). Acta Cryst. D53, 240-255.

Otwinowski, Z. (1991). Proceedings of the CCP4 Study Weekend. Isomorphous Replacement and Anomalous Scattering, edited by W. Wolf, P. R. Evans \& A. G. W. Leslie, pp. 80-86. Warrington: Daresbury Laboratory.

Otwinowski, Z. \& Minor, W. (1997). Methods Enzymol. 276, 307-326. Perrakis, A., Morris, R. M. \& Lamzin, V. S. (1999). Nature Struct. Biol. 6, 458-463.

Schröder, E., Littlechild, J. A., Lebedev, A. A., Errington, N., Vagin, A. A. \& Isupov, M. N. (2000). Structure. 8, 605-615.

Schröder, E. \& Ponting, C. P. (1998). Protein Sci. 7, 2465-2468.

Seo, M. S., Kang, S. W., Kim, K., Baines, I. C., Lee, T. H. \& Rhee, S. G. (2000). J. Biol. Chem. 275, 20346-20354.

Sheldrick, G. M., Dauter, Z., Wilson, K. S., Hope, H. \& Sieker, L. C. (1993). Acta Cryst. D49, 18-23.

Yeates, T. O. (1997). Methods Enzymol. 276, 344-358. 